\title{
The change in the energy metabolism of broiler chickens under the influence of Enterococcus faecium ICIS 96
}

\author{
Elena Kochkina ${ }^{1,{ }^{*}, \text { Alexey Torshkov }}{ }^{1}$, Larisa Kislinskaya $^{1}$, Olga Kartashova ${ }^{1,2}$, and Maria \\ Sycheva ${ }^{1,2}$ \\ ${ }^{1}$ Orenburg State Agrarian University, 460014, 18, Chelyuskintsev str., Orenburg, Russia \\ ${ }^{2}$ Institute of cellular and intracellular symbiosis UrD RAS, 460001, 11, Pionerskaya str., Orenburg, \\ Russia.
}

\begin{abstract}
The intensive development of domestic poultry farming determines the urgent need to achieve the environmental safety of the industry's products, which excludes the introduction of feed antibiotics into the poultry diet. In addition, the existing instability in the market for domestic biological products, whose share is currently no more than $40 \%$ and continues to decline steadily, dictates the need to search for approaches to import substitution. An example of a current trend in poultry farming is the use of domestic veterinary drugs of natural origin, which include probiotic feed additives containing cultures of live lactic acid microorganisms, typical representatives of which are enterococci. A wide search for bio-technologically valuable microorganisms among representatives of the genus Enterococcus allowed us to isolate and characterize the Enterococcus faecium ICIS 96 strain, which is promising for creating a probiotic feed additive. It is known from the literature that lactic acid microorganisms are able to regulate the metabolism of poultry. It is appropriate to assume that the culture of E. faecium ICIS 96, when introduced into the diet of poultry, can have a beneficial effect on energy metabolism. Clarification of this assumption determined the purpose of our work.
\end{abstract}

\section{Introduction}

The poultry industry is one of the most intensive and dynamic sectors of the agroindustrial complex in many countries and in the future, a further increase in the production and consumption of poultry products is expected. Industrial poultry farming has a significant potential for increasing the production of valuable food products in a short time [1]. It is possible to obtain high indicators of productivity and product quality only from healthy poultry, therefore, stable development of the industry is impossible without epizootic well-being.

For more than 50 years in the poultry industry, subtherapeutic doses of imported antibiotics have been used to prevent infectious diseases and increase the productivity of

\footnotetext{
${ }^{*}$ Corresponding author: eekochkina@gmail.com
} 
poultry. Recently, however, the use of feed antibiotics has been banned in many countries, since this not only creates a threat of the emergence and spread of antibiotic-resistant strains of microorganisms, contributes to the development of dysbacteriosis, metabolic disorders in poultry, but also leads to the contamination of livestock products with residual amounts of antimicrobial drugs [2].

To increase the yield of marketable products per unit area and reduce the fattening time in poultry farming all over the world, various feed additives are used [3-6]. However, the existing instability in the market of domestic biological products, whose share is currently no more than $40 \%$ and continues to decline steadily, dictates the need to search for approaches to import substitution. An example of the current trend in poultry farming is the use of domestic veterinary preparations of natural origin, which include probiotic feed additives containing cultures of live lactic acid microorganisms, typical representatives of which are enterococci.

Enterococci are typical lactic acid microorganisms, representatives of the symbiotic microbiota of the digestive tract of birds and mammals. Non-pathogenic variants of enterococci are involved in the colonization resistance of the biotope, producing a number of biologically active substances, including bacteriocins. Bacteria of this genus, showing antagonistic activity against a number of pathogens and not having virulence factors, are used as a component of biological products. Currently, there are no domestic probiotic preparations based on microorganisms of the genus Enterococcus.

Currently, enterococci are actively studied by scientists around the world [7]. The search for avirulent strains of bacteria of the genus Enterococcus is being actively pursued.

It is known that enterococci have useful properties, such as antagonistic activity against a wide range of pathogenic and opportunistic microorganisms, detoxifying, immunomodulatory functions, synthesis of organic acids, vitamins and other biologically active compounds, normalization of carbohydrates, bile acids, cholesterol in the body, restoration of the balance of intestinal microflora [8].

Microorganisms of the genus Enterococcus as growth stimulants in poultry diets began to be used relatively recently, but it has already been established through what mechanisms the active growth of poultry occurs. They contribute to the maintenance and restoration of the normal balance of microflora, which in turn leads to increased absorption of nutrients, and hence - to the active growth of the bird, and also have immunomodulatory activity [9].

Also, in numerous works of scientists, the antagonistic effect of enterococci in relation to pathogenic and opportunistic bacteria is described. Thus, E. faecium LCW 44 and $E$. durans 6HL strains synthesize antimicrobial peptides (enterocins) that have high antagonistic activity against a number of gram-positive and gram-negative bacteria [10].

According to Y. Wu et al. (2019), the probiotic strain E. faecium has a positive effect on broiler performance by increasing weight gain and reducing feed conversion. In addition, the use of this strain for birds helps prevent diseases, stimulate growth, and increase egg production [11].

As a result of a wide search for biotechnologically valuable crops among the representatives of this genus, we managed to isolate the culture Enterococcus faecium ICIS 96, a detailed description of which is presented in our work [12]. The strain was deposited in the Departmental collection of useful microorganisms for agricultural purposes of the DAS RAS as a promising one for inclusion in the composition of a probiotic feed additive. Some biological effects of E. faecium ICIS 96 culture in vivo on a model of broiler chickens have been evaluated. Meanwhile, it is not known how the energy metabolism of a bird changes when a strain of enterococcus is introduced into the diet.

All of the above predetermined the purpose of the study - to characterize the influence of the E. faecium ICIS 96 culture on the state of energy metabolism in broiler chickens. 


\section{Materials and Methods}

The research was carried out at the Department of Microbiology and Infectious Diseases and in the interdepartmental complex analytical laboratory of the Faculty of Veterinary Medicine of the Federal State Budgetary Educational Institution of Higher Education "Orenburg State Agrarian University". The experiments were carried out on 60 broiler chickens of the Cobb-500 cross. All experiments with animals were carried out in accordance with the ethical principles and regulations recommended by the European Science Foundation (ESF) and the Declaration of Helsinki.

A suspension of $E$. faecium ICIS 96 culture in sterile isotonic sodium chloride solution with a cell concentration of $1 \times 10^{9}$ in $1 \mathrm{ml}$ was added daily to the birds of the experimental group at the rate of $0.1 \mathrm{ml}$ of suspension per $1 \mathrm{~kg}$ of live weight. The control group of chickens remained intact. Poultry were fed with dry balanced compound feed with nutritional parameters corresponding to the recommended feeding standards of the Federal State Budget Scientific Institution Federal Scientific Center "All-Russian Research and Technological Poultry Institute" of Russian Academy of Sciences (FSC ARRTPI RAS). The bird had free access to food and water.

The experimental period in broiler chickens lasted 6 weeks, during which at 10, 20, 30 and 40 days of age, the birds took blood samples from the axillary vein.

In blood serum obtained after retraction of a blood clot, biochemical parameters were studied using a STAT FAX 1904 photometer (Awareness Technology, USA) using Olvex Diagnostic kits according to the instructions attached to them: glucose - by enzymatic colorimetric method without de-proteinization, pyruvic acid (PVC) - by enzymatic UVmethod, total cholesterol and triacylglycerols - by enzymatic colorimetric method, highdensity lipoproteins - by enzymatic colorimetric method.

In the course of the study, the live weight of the chickens of the control and experimental groups was taken into account by weighing on a scale. To analyze the growth of young poultry, a derived value was used - the average daily gain.

The numerical materials obtained in the course of the research were processed statistically with the determination of the mean values, the mean square deviation and the mean error of the mean. The significance of the differences between the compared indicators was assessed by the Student's t-test. Statistical data processing was carried out using an automated program Microsoft Office Excel 2007.

\section{Results}

Carbohydrate metabolism plays an important role in determining the level and intensity of other metabolism and is key in the energy exchange of the bird's body, since most cells, tissues and organs are evolutionarily adapted to cover their energy costs due to the oxidative decay of exogenous or endogenous carbohydrates [13].

The main indicator of carbohydrate metabolism is the concentration of sugar in the blood, mainly glucose, which is an important, though not the only source of energy [14]. 
$\mathrm{mmol} / \mathrm{l}$

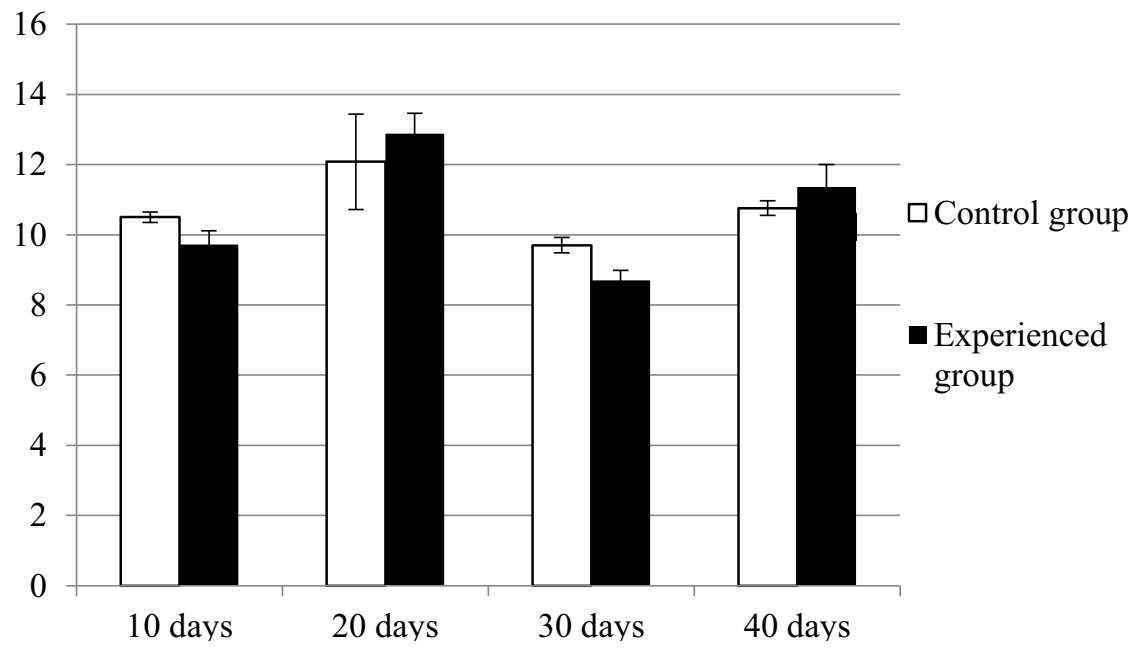

Fig. 1. Serum glucose concentration in broiler chickens

The results of our research showed that the concentration of glucose in the blood of broilers was not unambiguously dependent on the use of E. faecium ICIS 96 culture in the diet. At the age of 10 and 30 days, it was higher in broilers of the control group by 7.43$10.31 \%$, and at 20 and 40 days - in the experimental group by 5.58-6.62\%. At the same time, a general tendency of age-related changes in blood glucose concentration in broilers of both experimental groups was established: an increase in concentration during the second and fourth decades of post-incubation ontogenesis and a decrease in the period from 20 to 30 days of age, which looks quite logical, given the high rate of absolute and the relative growth of live weight at this age. Note that in the control group these changes were at the level of $10.93-19.70 \%$, while in the experimental group - 30.57-32.51\% and were statistically significant $(\mathrm{p}<0.001)$ (Fig. 1).

The main biochemical processes that are directly related to cellular energy exchange are the tricarboxylic acid cycle, fatty acid oxidation, carnitine cycle, electron transport in the respiratory chain, and oxidative phosphorylation. Since lactic and pyruvic acids are key metabolites of energy supply, they can be criteria for assessing energy homeostasis. Lactic acid is the product of anaerobic glycolysis, and pyruvic acid is necessary for tissue metabolism, as a result of which carbohydrates, "burning" in the tricarboxylic acid cycle, act as an energy substrate.

The study of the peculiarities of using the culture of enterococcus when growing broiler chickens showed that the concentration of pyruvic acid in the blood of poultry of the experimental group up to 30 days of age was higher than in intact chickens by $0.63-9.45 \%$. It was found that in the experimental group, age-related changes were characterized by an increase in the concentration of pyruvic acid by $2.98-17.53 \%$, while in the control group, a decrease by $3.89 \%$ by the age of 30 was noted (Fig. 2). 
$\mathrm{mmol} / \mathrm{l}$

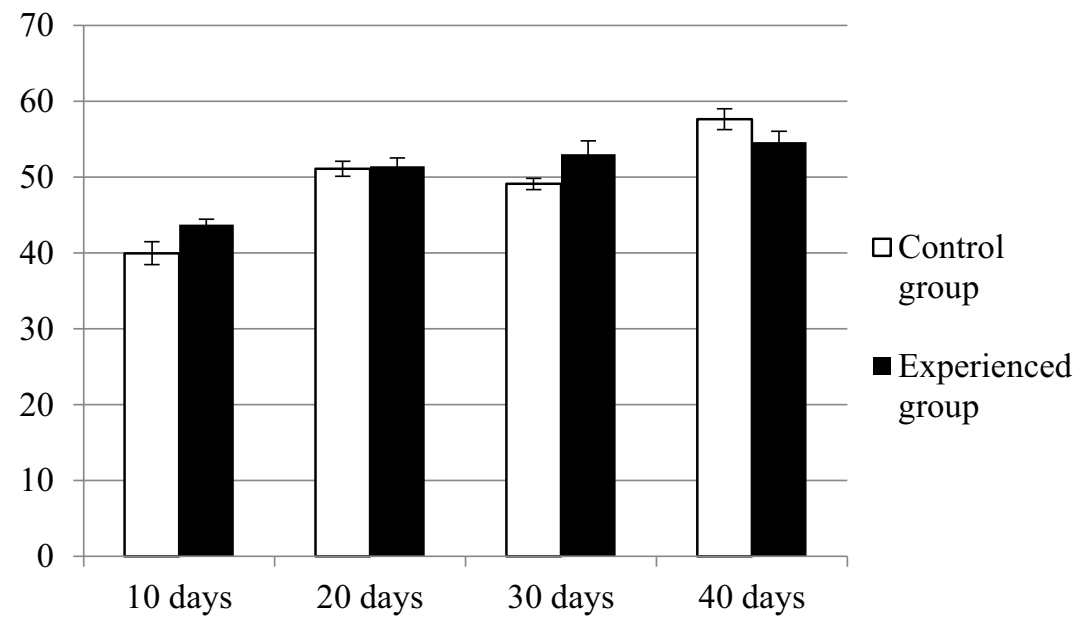

Fig. 2. Concentration of pyruvic acid in the blood serum of broiler chickens

A high metabolic supply with a source of readily available energy is a characteristic feature of fast-growing animals, and broiler chickens of modern crosses can be considered as such. Glucose is the main energy resource of the body, providing, in the presence of the proper amount of oxygen, the timely synthesis of the required amount of high-energy compounds serving protein synthesis and the functioning of striated muscles. However, the energy metabolic demand of broiler chickens is so high that the carbohydrate complex does not fully provide the plastic processes with energy at the cellular and subcellular levels. As a result, lipids are involved in energy metabolism, which are included in energy supply directly or through gluconeogenesis.

The state of lipid metabolism in general terms can be judged by the picture of blood lipids due to the fact that blood performs only the functions of transporting fatty substances, and oxidation and decay of lipids in it does not occur.

In the study of lipid metabolism, it is of great interest to determine the content of total cholesterol and lipoproteins in the blood. Our studies have shown that in the control group of broilers there was a general tendency towards a decrease in total cholesterol with age, with the achievement of minimum values by the end of the experimental period. At the same time, changes during the second and fourth decades of post-incubation ontogenesis were statistically significant $(\mathrm{p}<0.01)$. In the experimental group, a decrease in the concentration of total cholesterol in the blood occurs up to 30 days of age, reaching the minimum values, which are inferior to the control values on average $17.8 \%(\mathrm{p}<0.01)$ (Fig. $3)$.

Since lipids are insoluble in water, lipids resynthesized and newly synthesized in the intestinal wall do not enter the blood in their pure form. The problem of their transport in the blood plasma is solved by the interaction of non-polar lipids (triacylglycerols and cholesterol esters) with amphipathic lipids (phospholipids and cholesterol) and proteins, as a result of which lipoproteins that are miscible with water are formed, which, depending on the behavior in the gravitational field during ultracentrifugation, subdivide separate fractions: chylomicrons, very low density lipoproteins, low density lipoproteins and high density lipoproteins. 
$\mathrm{mmol} / 1$

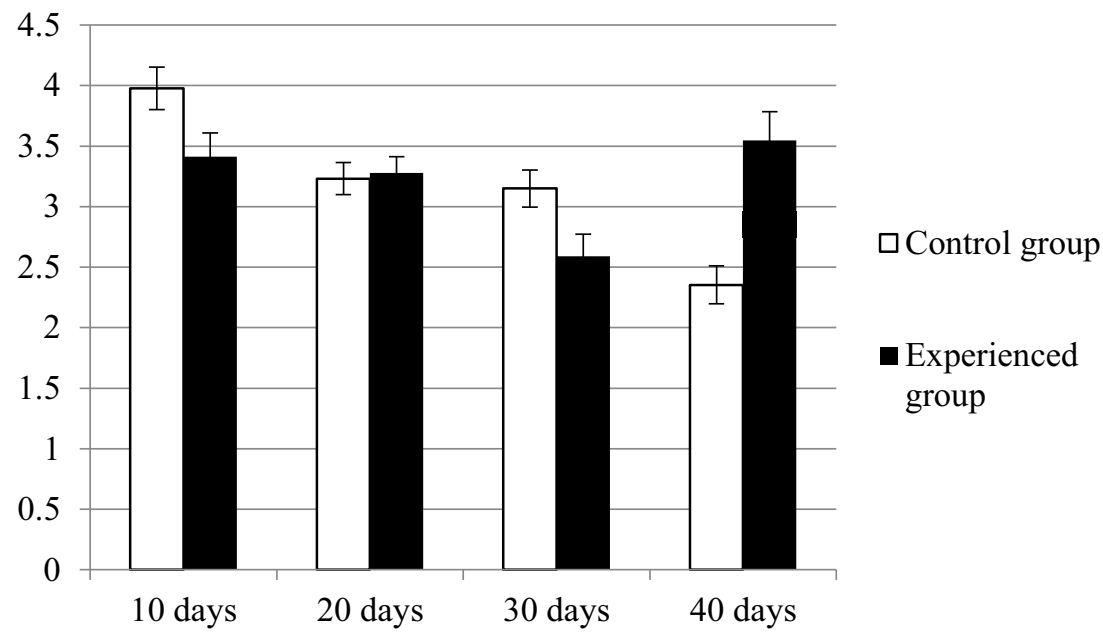

Fig. 3. Serum cholesterol concentration in broiler chickens

The content of cholesterols of lipoprotein fractions of various densities is one of the most important diagnostic tests of the state of the antioxidant-antiradical system, criteria for the state of metabolic processes in the body. This confidence is based on the actual status quo, according to which, the higher the functional activity of the antioxidant system, the lower the concentration of low density lipoprotein cholesterol (atherogenic factor) and the higher the concentration of high density lipoprotein cholesterol (antiatherogenic factor). The results of our research show that in broilers fed a culture of Enterococcus, in the period from 20 to 40 days of age, the concentration of high-density lipoprotein cholesterol increases by $24.4-67.4 \%$ compared to the analogs of the control group. The maximum difference in the concentration of high-density lipoprotein cholesterol between the birds of the control and experimental groups reaches at the age of 40 days $(\mathrm{p}<0.01)$ (Fig. 4).

$\mathrm{mmol} / 1$

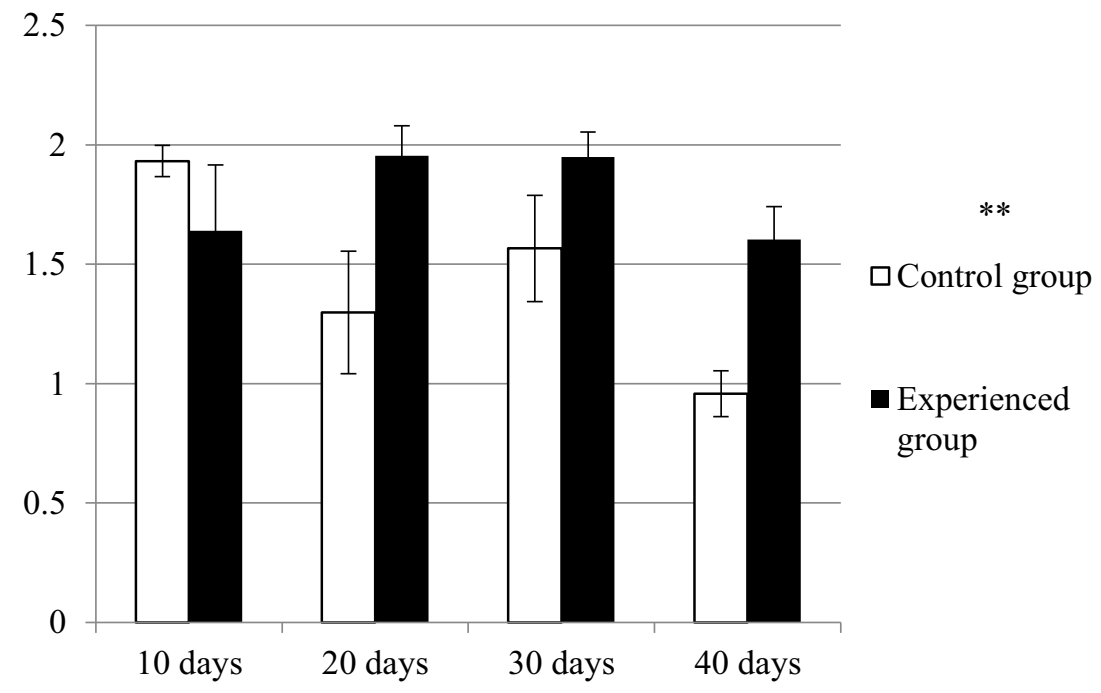

Fig. 4. Concentration concentration of high density lipoprotein cholesterol in serum of broiler chickens 
Triacylglycerols play an important role in energy metabolism in birds. Specific agerelated changes can be traced by the concentration of triacylglycerols in the blood as the basis for the intensive growth and development of broiler chickens. A decrease in the concentration of triacylglycerols in the blood of broilers of the experimental groups up to 30 days of age was established by $40.8-63.1 \%$ in the control and by $25.0-47.8 \%$ in the experiment, followed by an increase by $150.7 \%$ to 40 days of ageand $148.8 \%$, respectively. At the same time, if up to 20 days of post-incubation ontogenesis, the concentration of triacylglycerols in the blood of broilers that received enterococcus in the diet was inferior to that of chickens in the control group by $20.7-37.4 \%$, then at the last stages of the experiment, the prevalence of values in the experimental group was $11.3-12.2 \%$.

The above biochemical changes predetermined an increase in the productivity of broiler chickens fed with $E$. faecium ICIS 96 culture as part of the diet. By the age of 20 , the birds of the experimental group reach the maximum superiority over the control ones in terms of productivity. The average daily gain in live weight in the experimental group increased by $14.93 \%$ compared to the control, which led to a superiority in live weight by $13.31 \%$. By the end of the experimental period, the broilers of the experimental group were ahead of their intact peers in average daily gain by $10.47 \%$, and in live weight - by $10.14 \%$, respectively.

\section{Discussion}

In recent years, much attention has been paid to the search for avirulent strains of microorganisms that are promising for the creation of preparations for poultry farming on their basis, which would affect the increase in productivity and growth. By some indicators of energy metabolism, one can understand the effectiveness of the use of feed additives.

Blood glucose homeostasis is maintained through a combination of the following processes: digestion of feed carbohydrates, liver glycogen metabolism and glucose synthesis from various non-carbohydrate precursors (gluconeogenesis) [15]. Poultry has a higher blood glucose level than farm animals. This, on the one hand, testifies to the active use of carbohydrate substrates in the body of a bird as an energy raw material, which ensures a high intensity of metabolic reactions in the cells of organs and tissues. Probably, this feature is fixed in birds evolutionarily, because only the catabolism of carbohydrates makes it possible to quickly obtain the required amount of energy. On the other hand, it is about the fitness of body cells to use carbohydrate metabolites in the reactions of biosynthesis of protein and lipid components.

We believe that the use of E. faecium ICIS 96 in the diet of broiler chickens did not have an unambiguous relationship, but, in general, had a positive effect on the blood glucose concentration of the experimental bird.

In this study, feeding broiler chickens with E. faecium ICIS 96 increased serum pyruvic acid. Since pyruvic acid is a central metabolite and a connecting link between the metabolism of proteins, carbohydrates and fats, while simultaneously performing a regulatory function [16], the changes recorded in this experiment, in our opinion, should be assessed as positive.

Of particular interest is the study of lipid metabolism, in particular, the determination of the content of total cholesterol and lipoproteins in the blood. Cholesterol is contained in lipoproteins either in free form or in the form of esters with long-chain fatty acids. It is synthesized in many tissues from acetyl coenzyme A and excreted by bile in the form of free cholesterol or bile salts. Up to $80 \%$ of the body's cholesterol is synthesized in the liver, $10 \%$ - in the wall of the small intestine [17].

It was found that the supplement with E. faecium ICIS 96 contributed to a decrease in the concentration of cholesterol in the blood serum, however, in the chickens of the control 
group, the concentration of cholesterol was lower. Probably, this effect may be due to the ability of lactic acid bacteria to incorporate cholesterol into cell membranes during growth, bind it in the small intestine, and inhibit enzymes responsible for cholesterogenesis [18, 19].

Blood lipoproteins are a unique transport form of lipids in the body. They carry out the transport of lipids of exogenous and endogenous origin, carry out the "capture" of excess cholesterol from the cells of peripheral tissues and its "reverse" transport to the liver for oxidation into bile acids and excretion in bile, in addition, lipoproteins transport fat-soluble vitamins, hormones and other biologically active substances [20].

An increase in the content of cholesterol inside cells due to its increased synthesis or activation of intracellular hydrolysis of cholesterol esters deposited in the cell leads to an acceleration of the outflow of cholesterol from the cell, which is carried out by capturing cholesterol from the cell membranes of peripheral tissues by high-density lipoproteins and its reverse transport, mainly to the liver.

Since there are no enzymes in the cells of animal tissues that could catalyze the oxidation of cholesterol with the rupture of the sterol nucleus, not to mention the oxidation of the entire cholesterol molecule to $\mathrm{CO}_{2}$ and $\mathrm{H}_{2} \mathrm{O}$, cholesterol catabolism occurs in the liver (oxidation of cholesterol to bile acids), in the adrenal glands and placenta ( the formation of corticoid hormones from cholesterol), in the testicular tissue and ovaries (the formation of steroid sex hormones). In this case, the main metabolic pathway for the elimination of cholesterol from the body is its oxidation into bile acids in hepatocytes of the liver [21].

It was found that in broilers fed E. faecium ICIS 96 with food, the concentration of high density lipoprotein cholesterol increased in comparison with broilers in the control group. Our results are consistent with the data of V. Tayeri et al. (2018), who noted an increase in the concentration of high-density lipoprotein cholesterol and a decrease in the concentration of low-density lipoprotein cholesterol in the serum of birds when a multicomponent probiotic preparation containing lactic acid microorganisms was introduced into their diet [22].

Our research is consistent with the data of Z.F. Zhang, I.H. Kim (2016), who found that the probiotic strain Enterococcus faecium DSM 7134 reduced total cholesterol and increased serum high-density lipoprotein cholesterol in laying hens [23].

Similar results were obtained by M. Capcarova et al. (2010), who noted that the introduction of the probiotic strain Enterococcus faecium M 74 into the diet of chickens contributed to a two-fold decrease in the concentration of total cholesterol and total lipids in the blood plasma, compared with the birds of the experimental group. However, supplementation with probiotics did not significantly affect bird body weight [24]. On the contrary, our results indicate an increase in the productivity of broilers fed with E. faecium ICIS 96. Similar results were obtained by J. W. Park et al. (2016), who found an increase in productivity in chickens with the introduction of the probiotic strain E. faecium [25].

Triacylglycerols play an important role in energy metabolism in birds. According to various authors, in the blood plasma of chickens, depending on the breed, age and physiological state, the level of total lipids can reach 6.2-8.9 g / 1, of which triacylglycerols - from 0.4 to $1.25 \mathrm{mmol} / 1(\mathrm{~mm} / \mathrm{l})$. Overfeeding has an adverse effect on blood lipid profile. Hyperlipidemias can cause liver disease, cholestasis, pancreatic disease, renal failure, obesity. It was noted that at the height of oviposition, the content of triacylglycerols in the blood increases. In critical periods of a bird's life, with a relative decrease in the concentration of protein and glucose in the blood, an increase in the content of triacylglycerols is observed, which confirms the unity of metabolism.

\section{Conclusions}


Thus, the use of E. faecium ICIS 96 culture in the diet promotes the activation of energy metabolism in the body of broiler chickens at the early stages of ontogenesis in comparison with poultry of the control group, leads to an intensification of carbohydrate and lipid metabolism. The high metabolic rate in the experimental group of poultry naturally provides an increase in broiler productivity by an average of $10.14-10.47 \%$, which opens up new opportunities for the use of enterococcus cultures as part of feed additives for poultry farming.

\section{References}

1. E. Rowe, M. S. Dawkins, S. G. Gebhardt-Henrich, Animals (Basel), A Systematic Review of Precision Livestock Farming in the Poultry Sector: Is Technology Focussed on Improving Bird Welfare?, 8(1), 4562 (2018) doi: 10.1038/s41598-018-22604-2

2. M. Goodarzi, S. Nanekarani, N. Landy, Asian Pacific Journal of Tropical Medicine, Effect of dietary supplementation with onion (Allium cepa L.) on performance, carcass traits and intestinal microflora composition in broiler chickens, 4, 297-301 (2014) doi: 10.1016/S2222-1808(14)60459-X

3. P. Shokryazdan, M. F. Jahromi, J. B. Liang, Y. W. Ho, Journal of the American College of Nutrition, Probiotics: From Isolation to Application, 36(8), 666-676 (2017) doi: 10.1080/07315724.2017.1337529

4. E. Angelakis, Microbial pathogenesis, Weight gain by gut microbiota manipulation in productive animals, 106, 162-170 (2017) doi: 10.1016/j.micpath.2016.11.002

5. I. Giannenas, E. Bonos, I. Skoufos, A. Tzora, I. Stylianaki, D. Lazari, A. Tsinas, E. Christaki, P. Florou-Paneri, British Poultry Science, Effect of herbal feed additives on performance parameters, intestinal microbiota, intestinal morphology and meat lipid oxidation of broiler chickens, 59(5), 545-553 (2018) doi: 10.1080/00071668.2018.1483577

6. U. Gadde, W. Kim, S. Oh, Hyun S. Lillehoj, Animal Health Research Reviews, Alternatives to antibiotics for maximizing growth performance and feed efficiency in poultry: a review, 18(1), 26-45 (2017) doi: 10.1017/S1466252316000207

7. Y. Nami, R. V. Bakhshayesh, H. M. Jalaly, H. Lotfi, S. Eslami, M. A. Hejazi, Frontiers in Microbiology, Probiotic Properties of Enterococcus Isolated From Artisanal Dairy Products, 10, 30-43, (2019), doi: 10.3389/fmicb.2019.00300

8. H. Hanchi, W. Mottawea, K. Sebei, R. Hammami, Frontiers in Microbiology, The Genus Enterococcus: Between Probiotic Potential and Safety Concerns. An Update, 9, 1-16 (2019) doi: 10.3389/fmicb.2018.01791

9. D. Song, Y. W. Wang, Z. X. Lu, W. W. Wang, H. J. Miao, H. Zhou, L. Wang, A. K. Li, Poultry Science, Effects of dietary supplementation of microencapsulated Enterococcus faecalis and the extract of Camellia oleifera seed on laying performance, egg quality, serum biochemical parameters, and cecal microflora diversity in laying hens, 7, 2880-2887 (2019) doi: 10.3382/ps/pez033

10. O. B. Braïek, S. Smaoui, Biomed Research International, Enterococci: Between Emerging Pathogens and Potential Probiotics, 1, 1-13 (2019) doi: $10.1155 / 2019 / 5938210$

11. Y. Wu, W. Zhen, Y. Geng, Z. Wang, Y. Guo, Poultry Science, Effects of dietary Enterococcus faecium NCIMB 11181 supplementation on growth performance and cellular and humoral immune responses in broiler chickens, 1, 150-163 (2019) doi: $10.3382 / \mathrm{ps} /$ pey368 
12. T. M. Pashkova, A. S. Vasilchenko, Y. A. Khlopko, E. E. Kochkina, O. L. Kartashova, M. V. Sycheva, Genome Announcements, Genome Sequence of Enterococcus faecium Strain ICIS 96 Demonstrating Intermicrobial Antagonism Associated with Bacteriocin Production, 6(10), (2018) doi: 10.1128/genomeA.00126-18

13. A. Zheng, J. Luo, K. Meng, J. Li, L. W. Bryden, W. Chang, S. Zhang, L. X. N Wang, G. Liu, B. Yao, BMC Genomics, Probiotic (Enterococcus faecium) induced responses of the hepatic proteome improves metabolic efficiency of broiler chickens (Gallus gallus), 17, 89 (2016) doi: 10.1186/s12864-016-2371-5

14. B. C. Mulukutla, A. Yongky, T. Le, D. G. Mashek, W.-S. Hu, Trends in Biotechnology, Regulation of Glucose Metabolism - A Perspective From Cell Bioprocessing, 34(8), 638-651 (2016) doi: 10.1016/j.tibtech.2016.04.012

15. T. J. Wester, G. Kraft, D. Dardevet, S. Polakof, I. Ortigues-Marty, D. Rémond, I. Savary-Auzeloux, Nutrition Research Reviews, Nutritional regulation of the anabolic fate of amino acids within the liver in mammals: concepts arising from in vivo studies, 28(1), 22-41 (2015) doi: 10.1017/S0954422415000013

16. N. Schormann, K. Hayden, P. Lee, S. Banerjee, D. Chattopadhyay, Protein Science, An overview of structure, function, and regulation of pyruvate kinases, 28(10), 1771-1784 (2019) doi: 10.1002/pro.3691

17. F. R. Maxfield, G. van Meer, Current Opinion in Cell Biology, Cholesterol, the central lipid of mammalian cells, 22(4), 422-429 (2010) doi: 10.1016/j.ceb.2010.05.004

18. L.-G. Ooi, M.-T. Liong, International Journal of Molecular Sciences, Cholesterollowering effects of probiotics and prebiotics: a review of in vivo and in vitro findings, 11, 2499-2522 (2010) doi: 10.3390/ijms11062499

19. X. Zhao, Y. Guo, S. Guo, J. Tan, Applied Microbiology and Biotechnology, Effects of Clostridium butyricum and Enterococcus faecium on growth performance, lipid metabolism, and cecal microbiota of broiler chickens, 97, 6477-6488 (2013) doi: 10.1007/s00253-013-4970-2

20. H. Yamamoto, T. Takada, Y. Yamanashi, M. Ogura, Y. Masuo, M. Harada-Shiba, H. Suzuki, Scientific Reports, VLDL/LDL acts as a drug carrier and regulates the transport and metabolism of drugs in the body, 7(1), 633 (2017) doi: 10.1038/ s41598-017-00685-9

21. C. Röhrl, H. Stangl, Wiener Medizinische Wochenschrift, Cholesterol metabolism physiological regulation and pathophysiological deregulation by the endoplasmic reticulum, 168(11), 280-285 (2018) doi: 10.1007/s10354-018-0626-2

22. V. Tayeri, A. Seidavi, L. Asadpour, C. J. C. Phillips, Veterinary Research Communications, A comparison of the effects of antibiotics, probiotics, synbiotics and prebiotics on the performance and carcass characteristics of broilers, 42(3), 195-207 (2018), doi: 10.1007/s11259-018-9724-2

23. Z. F. Zhang, I. H. Kim, Journal of Animal Science, Effects of probiotic supplementation in different energy and nutrient density diets on performance, egg quality, excreta microflora, excreta noxious gas emission, and serum cholesterol concentrations in laying hens, 91(10), 4781-4787 (2013) doi: 10.2527/jas.2013-6484

24. M. Capcarova, L. Chmelnicna, A. Kolesarova, P. Massanyi, J. Kovacik, British Poultry Science, Effects of Enterococcus faecium M 74 strain on selected blood and production parameters of laying hens, 51(5), 614-20 (2010) doi: 10.1080/00071668.2010.513961

25. J. W. Park, J. S. Jeong, S. I. Lee, I. H. Kim Poultry Science, Effect of dietary supplementation with a probiotic (Enterococcus faecium) on production performance, 
excreta microflora, ammonia emission, and nutrient utilization in ISA brown laying hens, 95(12), 2829-2835 (2016) doi: 10.3382/ps/pew241 